\title{
Philosophiques
}

\section{Des fourmis et des hommes : examen des prémisses darwiniennes dans la pensée de Benoît Dubreuil Benoît Dubreuil, Human Evolution and the Origins of Hierarchies}

\section{Frédéric Bouchard}

Volume 39, numéro 1, printemps 2012

URI : https://id.erudit.org/iderudit/1011620ar

DOI : https://doi.org/10.7202/1011620ar

Aller au sommaire du numéro

Éditeur(s)

Société de philosophie du Québec

ISSN

0316-2923 (imprimé)

1492-1391 (numérique)

Découvrir la revue

Citer ce document

Bouchard, F. (2012). Des fourmis et des hommes : examen des prémisses darwiniennes dans la pensée de Benoît Dubreuil / Benoît Dubreuil, Human Evolution and the Origins of Hierarchies. Philosophiques, 39(1), 265-270.

https://doi.org/10.7202/1011620ar d'utilisation que vous pouvez consulter en ligne. 


\title{
Des fourmis et des hommes: examen des prémisses darwiniennes dans la pensée de Benoît Dubreuil
}

\author{
FRÉDÉRIC BOUCHARD
}

Tout d'abord, je dois préciser qu'en tant que philosophe de la biologie je travaille très peu sur l'être humain. Ce choix reflète en partie une préférence méthodologique: l'épistémologie de la biologie est d'autant plus efficace si elle en arrive à l'humain à la fin de sa réflexion, et non au commencement. Il est risqué de commencer une réflexion sur la biologie en commençant par l'humain, car cela introduit inévitablement des distorsions et des biais anthropocentriques. Je souligne cela simplement pour expliquer mon ignorance de la paléoanthropologie exposée dans le livre de Benoît Dubreuil (20II), et non pas pour discréditer le projet de l'auteur qui ne cherche pas à offrir une épistémologie de la biologie, mais plutôt à développer une hypothèse naturaliste de l'évolution de certaines organisations sociales et politiques humaines.

Cependant, il reste pertinent d'examiner la théorie évolutionnaire sous-jacente au naturalisme proposé par Benoît Dubreuil, et ce seront donc les présupposés darwiniens généraux de l'argument du livre que je vais discuter ici, ainsi que les questions épistémologiques afférentes. J'identifierai ce que je vois être quatre tensions de l'argument, en ce qui a trait à son darwinisme implicite et explicite.

\section{L'argument est-il (vraiment) néodarwinien?}

Dans la description canonique de l'évolution selon Darwin, les organismes sont sélectionnés dans leur environnement, et, en réponse à cette sélection et à l'hérédité, les espèces évoluent. Au cours du $\mathrm{XX}^{\mathrm{e}}$ siècle, cette explication se raffina pour inclure le rôle des gènes. Dans une certaine interprétation néodarwinienne, le rôle des gènes fut mis à l'avant-plan du processus évolutif. Ainsi, on décrit les gènes comme étant sélectionnés à travers les organismes qu'ils créent. Cette caractérisation trouva son porte-étendard le plus connu en la personne de Richard Dawkins, éthologue, et son fameux "Gène égoïste» (1976). Un des grands avantages de focaliser l'explication évolutionnaire sur les gènes est qu'il permet de résoudre un mystère apparent de l'évolution, l'altruisme ${ }^{1}$. La sélection naturelle semble prédire le «chacun

1. Je dois souligner que je ne partage pas la conception dawkinsienne de l'évolution ou autre lecture néodarwinienne orthodoxe, mais m'y attarder constituerait une discussion parallèle beaucoup trop longue (voir Bouchard 20II). 
pour soi » dans le combat pour la survie, alors comment expliquer le sacrifice d'un organisme pour un autre? En fait, le sacrifice d'un organisme pour un autre n'est plus un mystère si on reconnaît que deux organismes apparentés partageront plusieurs gènes identiques. Du point de vue égoïste du gène (ici la notion de point de vue et d'égoïsme sont bien sûr métaphoriques) il est rationnel de sacrifier un de ses porteurs (c.-à-d. un organisme) si cela permet à un autre porteur du même gène de survivre et se reproduire. Ainsi, le degré de parenté entre deux organismes permet de prédire la possibilité d'émergence de comportements altruistes et de sacrifice. Cette conception de l'évolution de l'altruisme est nommée sélection de parentèle (kin selection). Or cette conception permet difficilement d'expliquer l'altruisme dans de grands groupes où la parenté est éloignée et où la plupart des interactions se font avec des inconnus. Cela explique l'attrait pour certains (par ex. D.S. Wilson et E. Sober, I998) de perspectives de «sélection de groupe» (group selection) qui tentent d'expliquer l'évolution de l'altruisme non pas par l'action de gènes égoïstes, mais en examinant le succès évolutionnaire de groupes de faible parenté qui ont une proportion importante d'altruistes. Un groupe devient en quelque sorte une entité émergente dont les organismes sont des parties. Dans certains contextes, les groupes ( «superindividus») qui ont une plus grande proportion d'altruistes survivent mieux que les groupes dont plus de membres sont égoïstes. La compréhension de l'évolution de l'altruisme est donc clivée entre les approches parentèle (kin) (foncièrement réductionnistes) et les approches de groupes (relativement émergentistes).

Étant donné l'importance de l'évolution de l'altruisme dans la structuration des groupes d'organismes, il aurait été important pour ce livre de se positionner de manière claire dans ce débat. Or, même si de diverses manières l'argumentaire suggère un arrimage fort à l'évolution par sélection naturelle, le livre de Benoît Dubreuil semble louvoyer sur cette question. À de multiples reprises, il semble ancrer son argument dans un néodarwinisme des plus classiques (à ne pas confondre avec le néoévolutionnisme mentionné dans le livre), un néodarwinisme que l'on pourrait qualifier de dawkinsien. Cela laisse présager l'importance de la sélection de parentèle dans l'argument général. Or Dubreuil ne traite pas du tout du rôle de la sélection de parentèle et de la sociobiologie décrivant son fonctionnement. Si ce silence est motivé par un désir de relativiser ce néodarwinisme standard, alors pourquoi ne pas parler des autres théories ayant traité des associations entre organismes, comme la sélection de groupe évoquée il y a un instant?

En fait, le problème vient peut-être du fait que, malgré les suggestions répétées qu'il est évolutionniste, l'argument est en fait, à bien des égards, psychologique. L'approche du livre, parce que centrée sur les motivations, et donc sur la psychologie, est fondamentalement individualiste (au sens d'individu humain) ce qui minimise implicitement la possibilité que les groupes puissent être des entités émergentes sujettes à la sélection. Ironiquement, ce plaidoyer psychologisant minimise aussi le rôle du gène, car les motivations 
individuelles peuvent être en conflit avec le bien du gène (tel que décrit dans la sélection de parentèle). Ainsi, on a ici un argument naturaliste d'affinité darwinienne qui s'intéresse aux associations humaines, mais qui ne discute pas des processus darwiniens invoqués habituellement pour expliquer l'apparition des premiers groupes d'entraide primitifs.

\section{L'argument est-il (vraiment) déterministe par rapport à l'effet de l'évolution?}

Il y a des indices clairs dans le livre que l'action humaine serait déterminée par des forces externes. Ainsi, Dubreuil décrit (p. I 5 I) comment la centralisation est inéluctable dans les groupes humains. Ceci présume une constance dans les pressions de sélection favorisant cette centralisation. Toutefois, il tempère cela en suggérant qu'il identifie des contraintes limitant l'éventail des résultats contingents plutôt que des tendances lourdes déterminant un résultat donné: "My own explanatory focus on human cognition and motivation helps explain general constraints on the evolution of societies but says nothing about the direction of evolution" (p. 212).

Mais cette affirmation déflationniste ne correspond pas aux prédictions rétrodictives qui parsèment le livre. Par exemple: "There is no doubt that the spectacular growth of human populations in urban environments since the Industrial Revolution has accelerated and will further accelerate the pace of natural selection» (p. I 35 ). Il présume ici que le monde est assez stable pour que l'évolution traite nos changements sociaux de manière répétée et constante. En d'autre mots, si les contraintes sont tellement fortes qu'elles ne permettent qu'un éventail restreint de résultats, il n'y pas de différence entre l'identification de contraintes et la prédiction forte d'un résultat donné.

Il y a un autre indice plus fort de déterminisme sous-jacent. L'auteur examine longuement la trajectoire évolutionnaire des primates non humains, ce qui suggère deux choses: I) il y a une certaine continuité évolutive entre ces primates et nous (ce qui est indéniable); 2) les adaptations cognitives de nos ancêtres ont évolué chez nous d'une manière à rendre certains types d'association politique plus probables. Or le deuxième point présume que les pressions de sélection ont été stables à travers le temps et entre les espèces. Cette prémisse ne semble pas toujours préservée dans le livre. En biologie évolutionnaire, une certaine constance des pressions de sélection est nécessaire pour le "perfectionnement» d'un trait en adaptation. Seule une constance des pressions de sélection peut espérer une constance dans la réponse (adaptative) à la sélection. Par exemple, pour qu'il y ait adaptation d'une proie à courir plus vite, il faut que ses prédateurs tuent en fonction de qui est le plus lent, et ce, de manière répétée. Si les prédateurs changeaient continuellement de manière de chasser, il ne pourrait pas y avoir de lente accumulation menant à l'adaptation d'une stratégie particulière chez la proie. Mais il est beaucoup plus ardu de présumer une constance des pressions de sélection pour des traits comportementaux d'espèces cognitivement 
complexes qui ont plus de flexibilité pour modifier et créer leurs comportements. En fait, c'est exactement ce que souligne Dubreuil dans son livre lorsqu'il présente l' "ecological flexibility» (section 2.5.I). Il écrit: "Our capacity to colonize new environments is so impressive that it becomes difficult to define exactly what the human niche is " (p. 68). En soulignant la "flexibilité » comportementale d'Homo sapiens, Dubreuil affaiblit peut-être sans le vouloir: r) la possibilité qu'il y ait un régime stable de pressions de sélection naturelle touchant nos comportements; 2) la possibilité d'une explication foncièrement darwinienne de l'évolution des groupes humains (en rendant l'organisme flexible, il réduit son assujettissement à des pressions de sélection); 3) l'inéluctabilité de certains types d'organisations sociales. Bref, j'ai de la difficulté à voir si, avec l'argument du livre, nous sommes dans la continuité évolutionnaire normale ou plutôt dans un régime où les fluctuations environnementales sont tellement grandes que l'évolution est «arrêtée » pour l'être humain. La présentation offerte est paradoxale en ce qu'elle se veut foncièrement naturaliste, mais offre à ses adversaires les indices d'une différence humaine qui pourrait justifier un antinaturalisme (question reprise dans mon quatrième point).

\section{L'argument est-il panglossien?}

Gould et Lewontin (1979), dans une critique implicite de la sociobiologie, montrent les dangers du panadaptationnisme: ils voient la biologie évolutionnaire (de l'époque) comme étant si obsédée par une pensée optimisante qu'elle n'envisage même pas d'autres explications qui ne seraient pas exprimées en termes de sélection et d'adaptation. Ce genre de panadaptationnisme heuristique serait risqué selon Gould et Lewontin, car il est souvent utilisé de manière infalsifiable; si une explication adaptative échoue, on adopte souvent une autre explication adaptative sans pour autant remettre en cause le cadre adaptatif lui-même. Je ne sais pas ce qu'ils feraient de l'approche du livre ici discuté. Nous n'avons pas affaire ici à un déterminisme biologique fort (ce qu'ils décriaient tant dans la sociobiologie de E. O. Wilson), mais l'auteur endosse une heuristique foncièrement optimisante. Dans un cadre de pensée optimaliste, tout trait est d'abord analysé comme étant bénéfique (et donc implicitement adaptatif).

L'endossement le plus clair de la pensée optimaliste est présenté dans le livre par la question des artefacts. La culture matérielle est d'autant plus attractive qu'elle est souvent le résultat d'optimalité de design. La forme d'un silex a été transformée, a "évolué" si vous voulez, en réponse aux besoins des utilisateurs. Or ces changements matériels, opérés par apprentissage, peuvent difficilement changer le génome de leurs porteurs: il est donc difficile de voir un lien causal bi-directionnel entre culture matérielle et évolution biologique. Bref, la culture matérielle peut rester instructive et être liée, comme le montre Dubreuil, à des stades d'évolution, mais en faire la pierre d'assise de notre anthropologie politique évoque un panadaptation- 
nisme qui s'est révélé problématique en biologie. L'auteur est conscient de ces débats méthodologiques (p. I06) et il le souligne explicitement: «[...] We do not know how much variation in material culture can be accounted for by cultural or ecological variations» (p. I I6), et surtout: "Some traits are most likely the outcome of neutral mutation and genetic drift [...]» (p. I I7), mais l'heurisique pan-adaptationniste est tout de même présentée comme une heuristique extrêmement féconde pour le projet général. De la même manière que l'auteur n'évoque pas les contraintes dans la fabrication des artefacts (par ex. porosité d'une pierre, friabilité, propriété chimique, etc.), mais traite ces derniers plutôt comme des artefacts finis et perfectionnés en fonction d'une utilité (plutôt que le résultat d'un développement contingent), il ne traite pas vraiment des contraintes dans l'évolution biologique. Ainsi, plusieurs des adaptations biologiques présumées sous-jacentes à certaines motivations dont il discute sont peut-être seulement le résultat du hasard biologique (par ex., par dérive génétique évoquée par l'auteur luimême). Si la contingence biologique est plus grande que présumée dans le livre (ce qui est très plausible), on doit aussi relativiser la présomption d'optimalité de l'évolution des hiérarchies.

\section{L'argument est-il spéciste?}

Reprenons une distinction suggérée dans le chapitre IV par l'auteur entre les lumpers et les splitters. Les lumpers voient une vertu épistémique à unifier des phénomènes apparemment hétérogènes par des principes ou des processus homogènes. Les splitters ont tendance à exploiter l'hétérogénéité des phénomènes pour arguer l'incommensurabilité des phénomènes et donc l'impossibilité d'offrir une théorie unificatrice. En tant qu'unificateur, l'auteur critique l'anthropologie d'une tradition plus culturaliste qui, non seulement rejette la possibilité de principes unificateurs entre les groupes humains (chaque communauté étant en quelque sorte unique), mais a fortiori ces mêmes anthropologues culturels (et autres chercheurs en sciences sociales) rejettent toute unification des théories avec des théories expliquant les comportements de nos espèces apparentées (c.-à-d les grands primates). L'argument du livre est manifestement du type lumper dans la mesure où il veut réintégrer certains mécanismes motivationnels dans l'histoire évolutive de notre espèce, mais surtout montrer comment ces mécanismes permettent (ou facilitent) certains types d'associations humaines. Le deuxième pas est bien sûr le plus ambitieux. Toutefois, il révèle ici le côté splitter de l'auteur. Une grande partie du livre présume de la spécificité de l'évolution de notre espèce par rapport à l'organisation sociale. Dubreuil souligne par exemple: "Several features that are unique to human psychology and culture» (p. 4). Pour l'auteur (comme ironiquement pour les anthropologues culturels), l'espèce humaine est donc spéciale et unique. Mais en affirmant la spécificité de l'espèce humaine, l'auteur affaiblit involontairement la plausibilité de l'explication naturaliste darwinienne à laquelle il essaie de s'arrimer. 
En d'autres mots, on pourrait dire que la première moitié du livre nous montre que tous les animaux, incluant Homo sapiens, sont "égaux» d'une manière théorique parce que tous les animaux sont le produit de l'évolution (la partie lumper du livre), alors que la deuxième moitié du livre, en soulignant la spécificité de l'être humain, suggère que certains animaux sont plus égaux que d'autres... L'être humain serait spécial (la partie splitter). Je me demande si, en ne parlant pas plus d'évolution, de sociobiologie, d'unité de sélection, de processus complexes de rétroaction organismes/environnement, comme dans le cas de la construction de niche, etc., l'argument du livre n'a pas en fait internalisé l'exceptionnalisme humain qu'il reproche à certains anthropologues. La différence est que l'auteur applique cet exceptionnalisme à toute l'espèce Homo sapiens plutôt qu'à des communautés culturelles données. L'argument du livre reste riche et convaincant, mais je ne sais pas s'il est aussi darwinien qu'on le suggère à divers endroits du projet.

Le livre reste, malgré mes critiques, absolument remarquable; il est d'une ampleur et d'une érudition louable, et le projet général mérite une considération sérieuse. C'est beaucoup demander, mais mon espoir est que l'auteur, en intégrant de manière plus soutenue la théorie de l'évolution, soit en mesure de nous écrire éventuellement un livre sur la philosophie politique naturaliste des fourmis.

\section{Bibliographie}

Bouchard, Frédéric. "Darwinism Without Populations: a More Inclusive Understanding of the "Survival of the Fittest" ", Studies in History and Philosophy of Science Part C: Studies in History and Philosophy of Biological and Biomedical Sciences 42, $\mathrm{n}^{\circ}$ I (March 20II), I06- I 4 .

Dawkins, Richard. The Selfish Gene, New York, Oxford University Press, 1976.

Dubreuil, Benoît. Human Evolution and the Origins of Hierarchies: The State of Nature, Cambridge University Press, 20 Io.

Gould, S. J. and R. C Lewontin. "The Spandrels of San Marco and the Panglossian Paradigm: a Critique of the Adaptationist Programme» Proceeding of the Royal Society of London. Series B, Biological Sciences 205, n $\mathrm{n}^{\circ}$ I I6I (I979), 58I-598.

Sober, Elliott, and David Sloan Wilson. Unto Others the Evolution and Psychology of Unselfish Behavior, Cambridge, Mass, Harvard University Press, 1998. 